\title{
Photon echo in the super-radiant light scattering from a Bose-Einstein condensate
}

\author{
N. Piovella, ${ }^{1}$ V. Beretta, ${ }^{1}$ G. R. M. Robb, ${ }^{2}$ and R. Bonifacio ${ }^{1}$ \\ ${ }^{1}$ Dipartimento di Fisica, Università Degli Studi di Milano and INFM, Via Celoria 16, Milano I-20133, Italy \\ ${ }^{2}$ Department of Physics, University of Strathclyde, Glasgow G4 ONG, Scotland
}

(Received 4 April 2003; published 11 August 2003)

\begin{abstract}
Super-radiant Rayleigh scattering from a Bose-Einstein condensate, driven by a single far-detuned laser beam, is strongly limited by dephasing due to inhomogeneous broadening. We demonstrate that the dephasing can be reversed applying a Bragg pulse of area $\pi$ after the first superradiant scattering, stimulating a superradiant echo and inducing a further transfer of atoms from the initial condensate to a condensate with relative momentum $2 \hbar k$ in the direction of the laser.
\end{abstract}

DOI: 10.1103/PhysRevA.68.021801

\section{INTRODUCTION}

The highly coherent nature of a Bose-Einstein condensate (BEC), now routinely produced in many laboratories, offers the possibility to study new effects induced by the radiation on the atomic motional state. Optical lattices, created by pairs of off-resonance counterpropagating laser beams, are used to manipulate in a precise and controlled way the atomic center-of-mass motion of $10^{6}$ of atoms in a macroscopic quantum superposition [1]. One of the most attractive features of such systems is the long coherence time, of fundamental importance for high precision spectroscopy [2] and for quantum information [3].

In general, the average response of a quantum system prepared in a superposition state (as, for instance, that obtained by the spatial overlap of two atomic wave packets) typically decays not only due to loss of coherence (as, for instance, that induced by spontaneous scattering of photons), but also due to dephasing resulting from inhomogeneous broadening, i.e., resulting from a distribution of momentum of the atoms in the wave packet. In many cases decoherence cannot be studied directly because inhomogeneous dephasing is the dominant process.

Recently, evidence of loss of coherence has been observed in super-radiant Rayleigh scattering from an elongated BoseEinstein condensate [4-6]. The effect observed in these experiments shows how the long coherent time of the condensate leads to strong correlations between successive scattering events. This effect is related to the collective atomic recoil laser (CARL) [7], first proposed for cold atoms in a thermal state and then extended to the case of atoms in a BEC [8-10]. In this latter case, the CARL appears to be a promising source of macroscopic entangled atom-atom and/or atom-photon systems [11]. In a one-dimensional (1D) description of the super-radiant Rayleigh scattering process, the photons of a single far-detuned laser beam are backscattered by the atoms of the condensate to form a super-radiant pulse, whereas the atoms recoil coherently with an extra momentum of $2 \hbar k$ resulting from the transfer of a photon from the pump laser to the super-radiant beam. In the ideal decoherence-free case, the process is sequential, with the atoms scattering identical super-radiant pulses each time they decrease their momentum by a discrete step of $2 \hbar k$. Instead, the experiment of Ref. [4] has shown that the sequence is limited to a few super-radiant scattering events, depending
PACS number(s): 03.75.-b, 42.50.Fx, 42.50.Vk

on the decoherence rate-to-gain ratio. The dephasing causing the decay of the matter-wave interference results mainly from Doppler and mean-field broadening.

In this paper, we show that the inhomogeneous dephasing, causing coherence decay in super-radiant scattering from a $\mathrm{BEC}$, is a reversible process. In fact, by applying a short Bragg pulse of area $\pi$ at a time $\Delta t$ after the emission of the first super-radiant pulse, then a super-radiant echo is observed after $2 \Delta t$, inducing a further transfer of atoms into the recoiling condensate. The analysis is carried out in two steps. First, we approximate the coupled Maxwell-GrossPitaevskii (MGP) equations, describing a 1D condensate interacting with the pump and scattered radiation fields, by a Maxwell-Bloch (MB) model with inhomogeneous broadening, where the atoms are described by only two motional states. Solving the MB model, we demonstrate the existence of the super-radiant echo. Second, we demonstrate that the same effect is also observed using the more general MGP model.

\section{A MODEL FOR THE SUPER-RADIANT RAYLEIGH SCATTERING}

When the laser is sufficiently detuned from the atomic resonance, it leaves the atoms in the internal ground state. Consequently, radiation pressure due to absorption and subsequent random incoherent, isotropic emission of a photon can be neglected. In this detuned regime, coherent scattering of the pump laser is the dominant process. The atoms interact with a laser beam of wave vector $\vec{k}$ and scatter photons of wave vector $\vec{k}_{s}$ recoiling with a momentum $\hbar \vec{q}=\hbar\left(\vec{k}-\vec{k}_{s}\right)$. The atoms, initially scattered randomly into various momentum states, interfere with the atoms in the original momentum state. This creates a matter-wave grating having the correct periodicity to further scatter the laser beam in the direction $\vec{k}_{s}$. In an elongated condensate, a preferential direction for the scattered photons emerges causing superradiant Rayleigh scattering. Both the matter-wave grating and the scattered light are coherently amplified [4]. In a similar way, if the emitted light is allowed to build up in a cavity, an optical laser is realized as in the CARL [7]. In our onedimensional geometry, the laser beam is directed along the major axis of the condensate. As a consequence, photons are backscattered with $\vec{k}_{s} \approx-\vec{k}$ and the atoms move away from 
the original condensate with a relative momentum $\hbar q$ $\approx 2 \hbar k$ in the direction of the laser beam. The self-consistent evolution of the matter-wave field $\Psi$ and of the single-mode scattered radiation amplitude $a$ can be approximately described by a Maxwell-Gross-Pitaevskii model:

$$
\begin{gathered}
i \frac{\partial \Psi}{\partial t}=-\omega_{r} \frac{\partial^{2} \Psi}{\partial \theta^{2}}-i g\left[a e^{i(\theta+\delta t)}-\text { c.c. }\right] \Psi \\
+\Omega_{0}(t) \sin \left(\theta+\delta_{0} t\right) \Psi+\beta N|\Psi|^{2} \Psi, \\
\frac{d a}{d t}=g N \int d \theta|\Psi|^{2} e^{-i(\theta+\delta t)}-\kappa a,
\end{gathered}
$$

where $\theta=2 k z, a=\left(\epsilon_{0} V / 2 \hbar \omega_{s}\right)^{1 / 2} E$ is the dimensionless electric-field amplitude of the scattered beam with frequency $\omega_{s}, \omega_{r}=\hbar(2 k)^{2} / 2 m$ is the two-photon recoil frequency, $g$ $=(\Omega / 2 \Delta)\left(\omega d^{2} / 2 \hbar \epsilon_{0} V\right)^{1 / 2}$ is the coupling constant, $\Omega$ is the Rabi frequency of the laser beam, detuned from the atomic resonance frequency $\omega_{0}$ by $\Delta=\omega-\omega_{0}, d=\hat{\epsilon} \cdot \vec{d}$ is the electric dipole moment of the atom along the polarization direction $\overrightarrow{\boldsymbol{\epsilon}}$ of the laser, $V$ is the volume of the condensate, $N$ is the total number of atoms in the condensate, $\delta=\omega-\omega_{s}$, and $\epsilon_{0}$ is the permittivity of the free space. The matter wave field is normalized such that $\int_{-\infty}^{+\infty} d \theta|\Psi|^{2}=1$. The third term on the right hand side of Eq. (1) is the effect of an optical lattice potential with a slowly varying Rabi frequency $\Omega_{0}(t)$ and a mismatch $\delta_{0}$ between the frequencies of the two counterpropagating laser beams forming the lattice. In Eq. (1), the term $\beta N|\Psi|^{2} \Psi$ describes the atom-atom interaction due to binary collisions, where $\beta=8 \pi \hbar k a_{s} / m \Sigma, a_{s}$ is the scattering length and $\Sigma$ is the condensate cross section. In the following analysis, this term will be neglected, assuming the limit $4 \pi \hbar a_{s} n_{s} / m \ll \omega_{r}$, where $n_{s}$ is the average atomic density. Equation (2) has been written in the "mean-field" limit, which models the propagation effects of the light by replacing the nonuniform electric field by an average value and by adding to the equation a damping term with decay constant $\kappa \approx c / 2 L$, where $L$ is the condensate length and $c$ is the speed of light in vacuum. This term describes the escape of photons from the interaction region in a time $L / c$.

We introduce the Fourier transform in $\theta$ of the atomic field

$$
\widetilde{\Psi}(\bar{p}, t)=\frac{1}{\sqrt{2 \pi}} \int_{-\infty}^{+\infty} d \theta \Psi(\theta, t) e^{-i \bar{p} \theta},
$$

where $\bar{p}=p_{z} / 2 \hbar k$ is the momentum normalized in units of the recoil momentum $2 \hbar k$. Writing Eqs. (1) and (2) in terms of $\widetilde{\Psi}(\bar{p}, t)$, we obtain

$$
\begin{aligned}
\frac{d \widetilde{\Psi}(\bar{p}, t)}{d t}= & -i \omega_{r} \bar{p}^{2} \widetilde{\Psi}(\bar{p}, t)+g\left[a^{*} e^{-i \delta t} \widetilde{\Psi}(\bar{p}+1, t)\right. \\
& \left.-a e^{i \delta t} \widetilde{\Psi}(\bar{p}-1, t)\right]+\frac{\Omega_{0}(t)}{2}\left[e^{-i \delta_{0} t} \widetilde{\Psi}(\bar{p}+1, t)\right. \\
& \left.-e^{i \delta_{0} t} \widetilde{\Psi}(\bar{p}-1, t)\right]
\end{aligned}
$$

$$
\frac{d a}{d t}=g N e^{-i \delta t} \int_{-\infty}^{+\infty} d p \widetilde{\Psi}^{*}(\bar{p}-1, t) \widetilde{\Psi}(\bar{p}, t)-\kappa a
$$

Let us assume that at $t=0$, the atomic wave packet has a narrow momentum distribution $W_{0}(\bar{p})=|\widetilde{\Psi}(\bar{p}, 0)|^{2}$ with average momentum $\bar{p}_{0}$ and width $\sigma_{p}^{-}$. The dephasing rate is of the order of $\omega_{r} \sigma_{p}^{-}$. With these initial conditions, superradiance occurs for $\omega_{r} \sigma_{p}^{-}<g^{2} N / \kappa \ll \kappa[12]$. This condition implies $\kappa \gg g \sqrt{N}$, so that the field variable $a$ follows the atomic variables adiabatically. Furthermore, if $\omega_{r}>g^{2} N / \kappa$, the atomic system behaves as a two-level system, spanned by the momentum states around $\bar{p}_{0}$ and $\bar{p}_{0}-1$. Combining these two conditions, we find that in order to describe the system as a two-level system evolving super-radiantly, it is necessary that $\sigma_{p}^{-}<1$. Introducing the polarization variable

$$
S(\bar{p}, t)=\frac{1}{W_{0}(\bar{p})}\{\widetilde{\Psi} *(\bar{p}-1, t) \widetilde{\Psi}(\bar{p}, t)\} e^{-i \omega_{r}\left(1-2 \bar{p}_{0}\right) t},
$$

and the population difference variable

$$
W(\bar{p}, t)=\frac{1}{W_{0}(\bar{p})}\left\{|\widetilde{\Psi}(\bar{p}, t)|^{2}-|\widetilde{\Psi}(\bar{p}-1, t)|^{2}\right\},
$$

neglecting the terms involving all the other transitions except that from the state around $\bar{p}_{0}$ to the state around $\bar{p}_{0}-1$, and assuming the frequency-matching (Bragg resonance) conditions, $\delta=\delta_{0}=\omega_{r}\left(1-2 \bar{p}_{0}\right)$, we obtain the following Maxwell-Bloch equations:

$$
\begin{gathered}
\frac{d S(\bar{p}, t)}{d t}=-2 i \omega_{r}\left(\bar{p}-\bar{p}_{0}\right) S(\bar{p}, t)+W(\bar{p}, t)\left(g a+\frac{\Omega_{0}(t)}{2}\right), \\
\frac{d W(\bar{p}, t)}{d t}=-2 g\left[a S^{*}(\bar{p}, t)+c . c .\right]-2 S(\bar{p}, t) \Omega_{0}(t), \\
\frac{d a}{d t}=g N \int_{-\infty}^{+\infty} d \bar{p} W_{0}(\bar{p}) S(\bar{p}, t)-\kappa a .
\end{gathered}
$$

\section{PHOTON ECHO}

We have solved Eqs. (8) and (10) numerically with initial conditions $S(\bar{p}, 0)=0, W(\bar{p}, 0)=1$ and a radiation seed $a(0)=0.01 \sqrt{N}$, in order to simulate start up from spontaneous emission. Figure 1 shows the photon flux per atom $|a|^{2} / N$, (a), and the populations of the initial (continuous line) and final (dashed line) momentum states, (b), vs $\omega_{r} t$ for $g \sqrt{2 N}=\omega_{r}$ and $\kappa=10 \omega_{r}$. We have assumed that the momentum distribution $W(\bar{p})$ has a Gaussian profile centered at $\bar{p}_{0}=0$, i.e., $W(\bar{p})=\left(1 / \sqrt{2 \pi} \sigma_{\bar{p}}^{-}\right) \exp \left(-\bar{p}^{2} / 2 \sigma_{\bar{p}}^{2}\right)$, with $\sigma_{\bar{p}}^{-}$ $=0.02$. The atoms emit a super-radiant pulse with the typical ringing observed in inhomogeneously broadened atomic system [13]. After the decay of the super-radiant pulse and when the populations have reached a steady-state value, we apply a short Bragg pulse to the system at time $t=600 / \omega_{r}$ of area $\pi$, i.e., $\int_{-\infty}^{\infty} d t \Omega_{0}(t)=\pi$, with Gaussian shape and duration $\delta t$ 

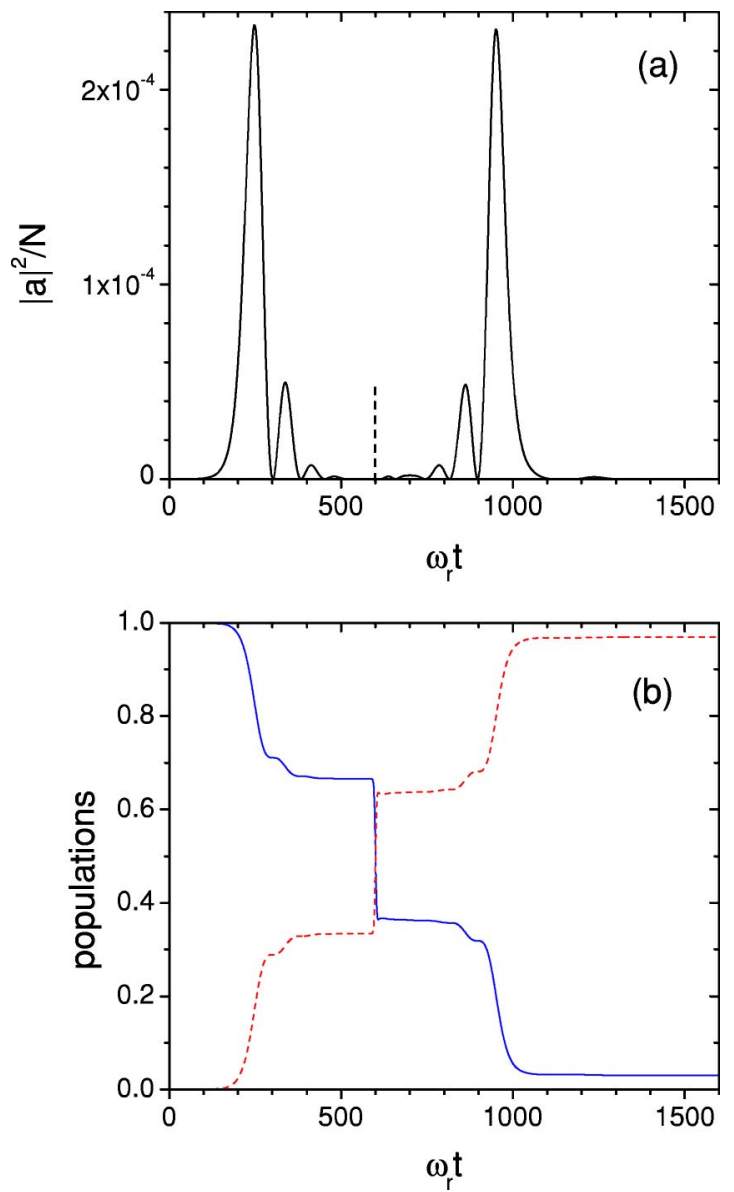

FIG. 1. Super-radiant echo as results from MB Eqs. (8)-(10), for $g \sqrt{2 N}=\omega_{r}, \kappa=10 \omega_{r}$, and $\sigma_{p}^{-}=0.02 ;|a|^{2} / N$ (a) and the populations of the initial (full line) and final (dashed line) momentum states (b) vs $\omega_{r} t$; the vertical dashed line in (a) marks the point where the $\pi$ pulse is applied.

$=2.5 / \omega_{r}$. vertical dashed line in Fig. 1(a)]. Note that during the application of the $\pi$ pulse, we have adiabatically switched off the pump laser (i.e., the coupling constant $g$ in our model) in order to avoid spurious superradiant emission during the population inversion process. After a time interval equal to that between the emission of the first super-radiant pulse and the $\pi$ pulse, we observe an echo of the superradiant field, reversed in time with respect to the former [Fig. $1(\mathrm{a})]$.

The populations of the two motional states [Fig. 1(b)] are only partially exchanged by the $\pi$ pulse. A complete population inversion could be obtained for a shorter $\pi$ pulse. However, a $\pi$ pulse which is too short has a larger bandwidth that may induce other transitions different from that involved in the super-radiant emission, i.e., from $\bar{p}=0$ to $\bar{p}$ $=-1$. The super-radiant echo does not depend on the duration of the Bragg pulse or on the population difference after the application of the pulse.

After the emission of the echo, the population of the initial state decreases by the same amount experienced during the emission of the first super-radiant pulse. We note that when the $\pi$ pulse completely inverts the populations of the two levels, the super-radiant echo completes the transfer of
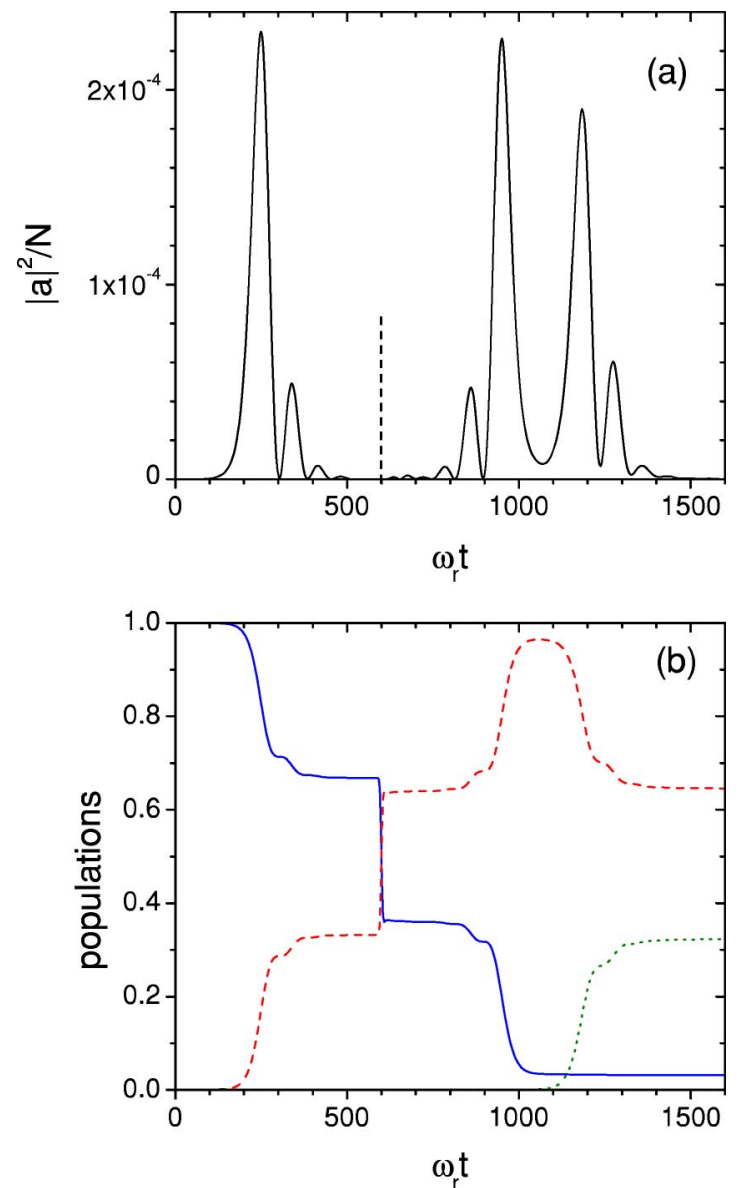

FIG. 2. Super echo as results from Eqs. (1) and (2), for $g \sqrt{2 N}$ $=\omega_{r}, \kappa=10 \omega_{r}$, and $\sigma_{p}^{-}=0.02$; (a) $|a|^{2} / N$ vs $\omega_{r} t$ (the vertical dashed line marks the point where the $\pi$ pulse is applied); (b) populations of the initial $\bar{p}=0$ (full line), $\bar{p}=-1$ (dashed line), and $\bar{p}=-2$ (dotted line) momentum states vs $\omega_{r} t$.

atoms from the initial to the final motional states. In fact, if $1-p$ is the fraction of atoms remaining in the initial condensate and $p$ is the fraction transferred to the scattered condensate after the emission of the super-radiant pulse, then, after the application of the $\pi$ pulse, the atomic fractions of the original and scattered condensates become $p$ and $1-p$, respectively. If the super-radiant echo is identical to the original pulse, it will transfer the same fraction of atoms from the initial to the scattered condensates, so that, when the $\pi$ pulse completely inverts the populations, the echo will transfer all the atoms to the scattered condensate. The small fraction of atoms remaining in the original condensate, visible in Fig. 1(b), is due to the incomplete population inversion produced by the $\pi$ pulse. The example above shows that in the reduced MB model, if there is no incoherent decay of the polarization, the dephasing is completely reversible. The echo effect could be used to measure the true coherence time of the condensate. In fact, the irreversible coherence decay can be taken into account by adding a damping term $-\gamma S$ to the right-hand side term of Eq. (8). The effect of this term is to decrease the transfer of the atoms from the initial state to the final state, leaving the fraction $2 \gamma / G$ of atoms in the initial state, where $G$ is the super-radiant gain. Then, measuring the 
stationary population fraction of the initial state after the super-radiant echo and $G$ from the rise time of the signal (which is inversely proportional to $G-2 \gamma$ ), it will be possible to measure the decoherence time $1 / \gamma$.

In order to demonstrate the possibility of producing a super-radiant echo using a condensate, as shown above by integrating the MB model, we performed a numerical simulation using the exact MGP model [Eqs. (1) and (2)], which were solved numerically using a split-step Fourier method [14]. The initial wave packet is still a Gaussian with $\sigma_{z}$ $=2 \lambda$, in order to have the same momentum spread of the case shown in Fig. 1. Using the same other parameters as were used for Fig. 1, the simulation produces the same radiation profile of the super-radiant pulse, shown in Fig. 2(a). We note that such a short longitudinal length for the wave packet should not justify the mean-field and single-mode approximations assumed for the scattered radiation field. However, a more realistic simulation using a condensate much longer than $\lambda$ would require an extremely long integration time. Nevertheless, the results of the simulation can be easily scaled to more realistic dimensions of the condensate, without invalidating the basic nature of the effect.

In order to obtain the population of the momentum state $\bar{p}=n$, we have integrated the momentum probability $|\widetilde{\psi}(\bar{p}, t)|^{2}$ over an interval centered at $\bar{p}=n$ and of unitary length. This can be done only if the momentum distribution remains narrower than the momentum level separation, i.e., if $\sigma_{p_{z}} \ll 2 \hbar k$. The result is shown in Fig. 2(b). At $t$ $=600 / \omega_{r}$, we apply the $\pi$ pulse, with a Gaussian shape and a duration $\delta t=2.5 / \omega_{r}$. As in the case of the MB model, the Bragg pulse cannot be made very short in order to avoid inducing other transitions different than that from $\bar{p}=0$ to $\bar{p}=-1$. This requires that the bandwidth of the $\pi$ pulse should be narrower than the recoil frequency, i.e., $\omega_{r} \delta t \gg 1$. The restored coherence between the atoms allows, after the superradiant echo, for a further transition from $\bar{p}=-1$ to $\bar{p}$ $=-2$, with the emission of another super-radiant pulse following the echo. This can be seen from Fig. 2(b), in which the population of the level $\bar{p}=-2$ is represented by the dotted line.

We observe that using the MGP model the echo is almost identical to the first super-radiant pulse, even if the population difference after the application of the $\pi$ pulse is less than before it. What is important in this case is the polarization between the states and the complete rephasing of the atoms. Because the atoms are all in phase after the echo, they are able to make another transition to $\bar{p}=-2$ and emit another super-radiant pulse, similar to the previous ones.

Finally, we give the parameters for a typical experiment which could be used to demonstrate the super-radiant echo. Let us consider a Bose-Einstein condensate of ${ }^{87} \mathrm{Rb}$ atoms driven by a laser detuned by $\Delta=(2 \pi) 10 \mathrm{GHz}$ from the $D_{2}$ line $(\lambda=780 \mathrm{~nm})$, with intensity $I=10 \mathrm{~mW} / \mathrm{cm}^{2}$. The atomic cloud contains $N=10^{6}$ atoms in a pencil-shape volume with longitudinal and transverse dimensions of $100 \mu \mathrm{m}$ and $10 \mu \mathrm{m}$, respectively, with density $n_{s}=10^{14} \mathrm{~cm}^{-3}$. The coupling constant is $g \sqrt{N} \approx 9.77 \times 10^{7} \mathrm{~s}^{-1}$ and the radiation loss $\kappa \approx 1.5 \times 10^{12} \mathrm{~s}^{-1}$, giving a super-radiant gain $G$ $=g^{2} N / \kappa \approx 6.4 \mathrm{~ms}^{-1}$. Because $G$ is less than the recoil frequency $\omega_{r}=95 \mathrm{~ms}^{-1}$, the condensate can be described approximately by two motional states with average momentum $p_{z}=0$ and $p_{z}=-2 \hbar k$. Assuming an overall momentum spread of $\sigma_{p}^{-}=0.027$ (in units of $2 \hbar k$ ) due to Doppler broadening and finite size of the wave packet, the coherence decay is of the order of $\omega_{r} \sigma_{p}^{-}=2.56 \mathrm{~ms}^{-1}$. With these parameters, a super-radiant emission similar to that shown in Fig. 1 should occur after $t=1.7 \mathrm{~ms}$. Applying a $\pi$ pulse of duration $20 \mu \mathrm{s}$ after $t=5 \mathrm{~ms}$, the echo should be visible after $t$ $=8.3 \mathrm{~ms}$.

\section{CONCLUSION}

In conclusion, we have given evidence of a new echo effect in the super-radiant Rayleigh scattering by a BoseEinstein condensate, obtained by applying a Bragg lattice pulse of area $\pi$ after the emission of the super-radiant pulse. This technique could allow measurement of the true coherence time of the condensate, isolating the coherence decay due to dephasing as a result of inhomogeneous momentum broadening of the atomic wave packet.
[1] See, for instance, Ph.W. Courteille, V.S. Bagnato, and V.I. Yukalov, Laser Phys. 11, 659 (2001).

[2] D.J. Wineland, J.J. Bollinger, W.M. Itano, F.L. Moore, and D.J. Heinzen, Phys. Rev. A 46, R6797 (1992).

[3] See, for example, The Physics of Quantum Information, edited by D. Bouwmeester, A. Ekert, and A. Zeilinger (Springer, Berlin, 2000).

[4] S. Inouye, A.P. Chikkatur, D.M. Stamper-Kurn, J. Stenger, D.E. Pritchard, and W. Ketterle, Science 285, 571 (1999).

[5] M. Kozuma, Y. Suzuki, Y. Torii, T. Sugiura, T. Kuga, E.W. Hagley, and L. Deng, Science 286, 2309 (1999); S. Inouye, T. Pfau, S. Gupta, A.P. Chikkatur, A. Gorlitz, D.E. Pritchard, and W. Ketterle, Nature (London) 402, 641 (1999).

[6] D. Schneble, Y. Torii, M. Boyd, E.W. Streed, D.E. Pritchard, and W. Ketterle, Science 300, 475 (2003).
[7] R. Bonifacio and L. De Salvo Souza, Nucl. Instrum. Methods Phys. Res. A 341, 360 (1994); R. Bonifacio, L. De Salvo Souza, L.M. Narducci, and E.J. D’Angelo, Phys. Rev. A 50, 1716 (1994).

[8] M.G. Moore and P. Meystre, Phys. Rev. A 58, 3248 (1998).

[9] M.G. Moore, O. Zobay and P. Meystre, Phys. Rev. A 60, 1491 (1999).

[10] N. Piovella, M. Gatelli, and R. Bonifacio, Opt. Commun. 194, 167 (2001).

[11] N. Piovella, M. Cola, and R. Bonifacio, Phys. Rev. A 67, 013817 (2003).

[12] R. Bonifacio and L.A. Lugiato, Phys. Rev. A 11, 1507 (1975).

[13] See, for instance, N. Skribanowitz et al., Phys. Rev. Lett. 30, 309 (1973).

[14] A. Newell and J. Moloney, Nonlinear Optics (Addison-Wesley, Redwood City, CA, 1992). 